\title{
Integrated Wide Area Protection and Control Systems based on Advanced Communication Network
}

\author{
Z. Q. BO, Shengming GE, Q. P. WANG, Lin WANG, Fengquan ZHOU, Xing LIU and Z. F. \\ FAN \\ XUJI Group, State Grid Corporation, Beijing, China
}

B M ZHANG

Department of Electrical Engineering, Tsinghua University, Beijing, China

\begin{abstract}
As a new protection, wide-area protection enables protective relaying, and automatic control based on electric power system network communications and comprehensive judgment of multi-point information, which plays an increasingly important role in the secure and stable operation of electric power system. The recent development in power system protection and control is firstly reviewed in this paper, with more concentration on the wide-area and integrated protection. Then the principle of integrated wide area protection and control is introduced, in which a hierarchical protection and control system provides the protection and control of wide area or regional power substations/plants and their associated power network. There are three levels in this system, which are the local, the substation/plant and the wide area/regional protection and control. The integrated functions at each level are described in details so as to develop an optimal coordination mechanism between the levels. Besides, This paper introduces the newest communication technology (Packet Transport Network), which can provide high speed and reliable communication network for integrate wide area protection and control. The network layer model and equipment architecture of PTN is presented in this paper, relevant analysis and discussion are stated in detail.

KEYWORDS: integrated protection; wide area protection; protection and control; packet transport network
\end{abstract}

\section{INTRODUCTION}

In recent years, the continuous development in communication technology and the possible to utilize fault generated transient for protection has resulted in the new relay protection principle to protect wide area power network [2, 3]. Immediately following the development, the concept of wide area protection mainly focused on control aspect has been presented [4]. The further development in both microprocessor and transducer technologies and the dramatic growth in signal processing power of relay platforms, and the availability of suitable communications schemes, have provided a new opportunity to revisit the concept of integrated protection. Research [5] shows that information obtained from multiple power plants and components can be used to derive new protection principles and schemes. Extensive research has been conducted on the implementation and application of integrated substation protection since. Recently the potential of the integration of protection and control for wide area application has also emerged [6].

Based on the above mentioned developments, this paper introduces a new concept of integrated wide area protection and control (IWAPC). The main focus of the concept is the integration between the protection and control, particularly at the wide area or regional level. Studies show that the IWAPC is able to provide a number of benefits to the future protection and control system with the potential to merger the three line of defense system, such as selfhealing on-line decision making, in order to prevent cascading tripping of large area power network.

\section{DEVELOPMENTS OF WIDE AREA AND INTEGRATED PROTECTION}

\subsection{Wide Area Protection}

In recent year, the fast development in communication technologies makes the wide area information exchange become possible. In this respect, the emergence of the wide area measurement system provides a new idea for the design of power system protection systems. The first wide area protection principle is derived from the transient based protection in 1996 [2], in which GPS time synchronization plays the major role in the design [3]. Immediately followed a summary paper in 1997 systematically outlines the concept of the so called 'wide area protection'[4], which mainly focus 
on the control aspect of the area. The wide area protection based on novel algorithms derived from the measurements of multiple points of information is able to provide fast, reliable and accurate fault clearance, and analyzes the effects on the system stability based on the fault system analysis and takes necessary control measures, to achieve the functions of relay protection and security and stability control to prevent voltage collapse The wide area relay protection has quickly become a hot research topic with many research results published particularly in recent years.

\subsection{Integrated Protection}

With the development of digital technology, more and more protection functions for an apparatus (line, transformer, generator etc.) have been implemented within one protective device to achieve a certain degree of integration. For example, a numeric line protection relay may have distance or current differential function as the main protection, and directional and over current functions, etc. as the backup protection. The dramatic growth in signal processing power of relay platforms, and the availability of suitable communications schemes in recent years, has provided a new opportunity to derive new protection principles and schemes based on the information obtained from multiple power plants and components, which could have significant advantages over the existing protection techniques based on individual plant or component. Unlike centralized (or substation area protection) the integrated protection does not simply centralize the relay hardware/software, mainly concentrates on the developments of new concepts and algorithms based on multiple points of measurements, it is hoped from which the performance of protection can be significantly improved.

\section{INTEGRATED WIDE AREA/REGIONAL PROTECTION AND CONTROL}

The proposed integrated wide area or regional protection and control system (IWAPC) is given in Figure.1. As shown, the latest developments in transmission system are mainly the series compensation in AC lines and high voltage DC lines; and in distribution system are mainly distributed generations and energy storage, etc. These new developments results in far more complicated characteristics than that of conventional systems, consequently the existing protection and control system will no longer effective to cope with the new systems, this has led to the proposed IWAPC system. As shown, the IWAPC system mainly consists of equipment at three layers: 1) the integrated multiple function intelligent equipment at the local (bay) level; 2) the integrated substation protection and control at the substation level and 3) the integrated wide area (regional) at wide area level (IWAPC). The key parts of the system are the high speed wide area communication network (PTN) and the real-time synchronization information platform of the IWAIP:

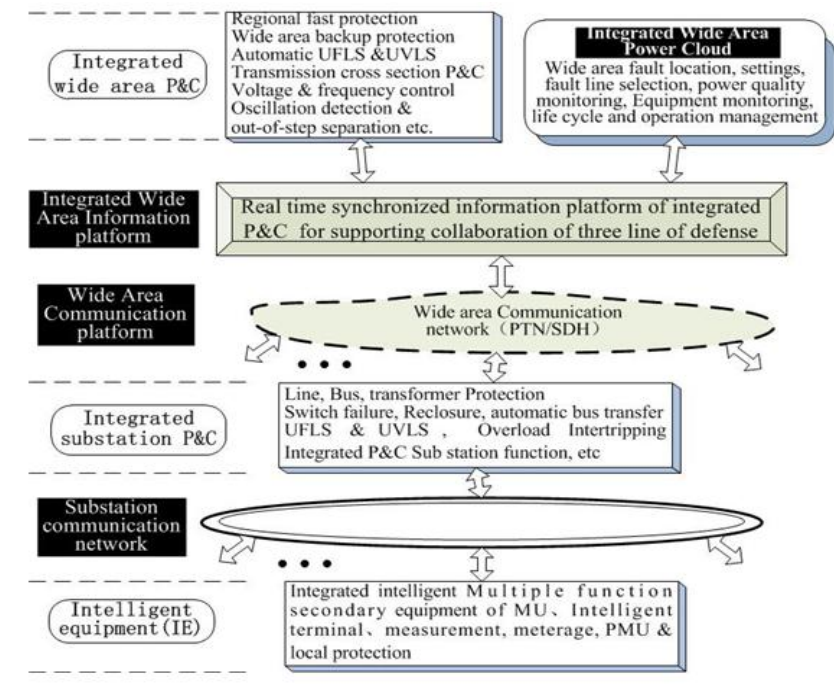

Figure.1 Integrated wide area protection and control

The IWAPC is extended to dispatching to realize the integration of dispatching automation, protection and control of power grid, and according to the three level dispatching (country, province, regional) architecture to implement the functions of regional protection, control and dispatching management.

\subsection{System Architecture}

Multiple functions intelligent equipment at the local level. As shown in Figure.1, the Intelligent equipment at local level is an integrated multiple function secondary equipment at each bay in the substation, which mainly consists of the MU, Intelligent terminal, measurement, metrology, PMU $\&$ local protection. The IEPC is responsible to sample all real-time data and send to integrated substation $\mathrm{P} \& \mathrm{C}$ and wide area $\mathrm{P} \& \mathrm{C}$, it also receives and carried out the control commands from the ISPC and IWAPC. The IEPC can be integrated into primary power apparatuses and achieve local protection for $90 \%$ of its associated line sections. It is redundant configuration with the integrated functions of fault recorder, data storage and network analysis, etc.

Integrated substation protection and control at the substation/plant level. The ISPC integrates functions of line, Bus, transformer protections; switch failure; auto reclosure; automatic bus transfer; UFLS, UVLS, Overload inter-tripping and substation control function, etc. It utilizes information from entire substation to achieve substation backup protection and safety automatic control etc. The CBs 
are used as a unit to configure the adaptive backup protection, and current differential protection is used to replace the stage over current protection, breaker failure protection and dead zone protection in the conventional protection system.

Integrated wide area/regional protection and control. The IWAPC specially designed for the protection and control of transmission network is equipped with the backup protection and the IRPC for distribution network is able to offer fast protection. In addition, they both integrate functions of automatic UFLS and UVLS, voltage and frequency control, oscillation detection and out-ofstep separation etc. In addition, the IWAPC also incorporates the function of transmission cross section safety $\mathrm{P} \& \mathrm{C}$. Unlike conventional protection and control, which are separated design and operation, the IWAPC integrated protection and control into one optimal combined system, which effectively coordinates the wide area (regional) protection and control, in order to achieve significant improvements in the protection and control of power systems.

\subsection{Synchronized High Speed Communication Network}

One of the most important elements of the IWAPC system is the fast communication network. The communication network proposed in here is the Packet Transport Network(PTN). The present power communication network is mainly used in multiservice transport platform based on the Synchronous Digital Hierarchy (SDH). Its advantages lie in high efficiency for carrying TDM services, low latency, high reliability, with end management capabilities. However, with the new trends in smart grid development, SDH technology gradually revealed its limitations, such as low bearing efficiency and poor flexibility for data services. By contrast, PTN can realize statistical multiplexing and efficient transfer of packet service by using Packet-switched core, which can overcome the weaknesses of SDH rigid bandwidth. In addition, it can provide good quality of service, operation, administration and maintenance. Self-healing fibre optical network is employed to connect to a number of substations in the region, to ensure the fully sharing of dynamic and transient information for all electrical measurements, breaker status and protection operations, high reliability IEEE-1588 technology to ensure the synchronization timing of the sharing data, to prove the data for the integrated wide area protection and control.

PTN technology is a integration of transport network technology and data network technology, which can carry several different operation such as Ethernet, TDM, ATM, IP/MPLS. At the same time, it can meet the demand of standardized operation, reliability, expand-ability, QoS and OAM. It is expected to replace traditional SDH/MSTP technology.

\subsubsection{Single Layer Network Model}

PTN is a packet-based and connection-oriented network technology. It combines the advantages of SDH and Ethernet and also enables end to end management features. Besides, its layer network model is similar to SDH, which includes Virtual Channel(VC) layer, Virtual Path(VP) layer, Virtual Segment(VS) layer and Physical Media layer. The PTN layer network model and the multiplexing relationships between layers will be shown in Figure. 2.

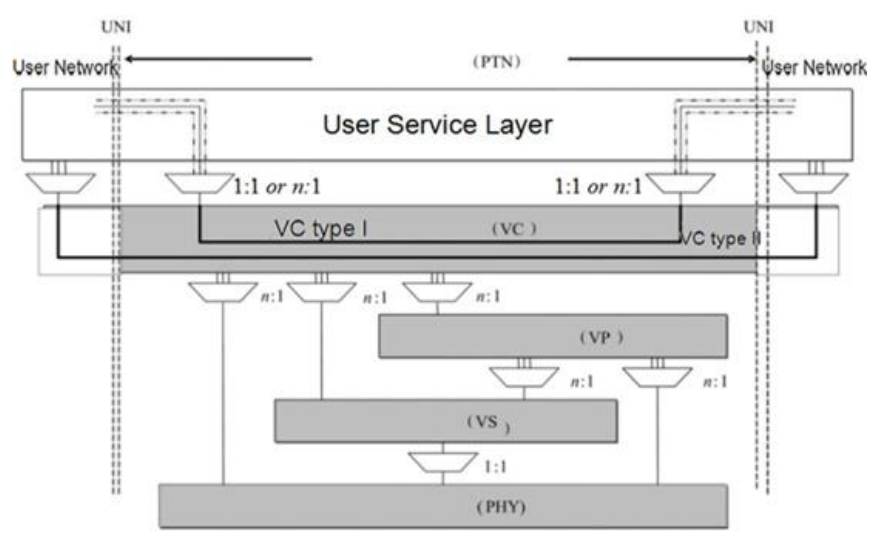

Figure.2 PTN layer network model and the multiplexing relationships between layers.

\section{- Virtual Channel(VC) layer}

For PTN based on MPLS-TP, VC layer is Pseudo Wire(PW) layer. The layer is similar to the LO-VC layer of $\mathrm{SDH}$, which can provide end to end transport network service for users. The VC layer will package user service content from User Service Layer and then the packaged content will be mapped to VP layer for bearing.

- Virtual Path(VP) layer

For PTN based on MPLS-TP, VP layer is LSP layer. It is equivalent to the tunnel layer of MPLS. VP layer is similar to the HO-VC layer of SDH, which can provide more transport network path for one user or many users. VP layer also support connection establishment and monitoring of the path. Besides, VP layer will provide adapter for the VS layer.

- Virtual Segment(VS) layer

The VS layer of PTN is similar to the segment of SDH. It is used for ensuring the entire link between adjacent nodes on the transport path. It can also bear the information from VP layer. Besides, VS layer can monitor the quality of link.

- Physical Media layer

Physical Media layer is used to realize the transport of bit-stream. Physical media could be optical media or electric media such as optical fiber, copper and etc. 
As is shown in Figure.2, the signal relationship between different PTN layers can be one to one or several to one, which looks like the mechanism of progressive multiplexing and transport of SDH. However, the multiplexing mechanism of PTN is actually Statistic Division Multiplex (SDM), Which is obviously different from the Time Division Multiplex (TDM) of SDH.

\subsubsection{Equipment Architecture}

The equipment architecture of PTN is similar to the equipment of MSTP based on SDH. It can be viewed as inheritance and development of MSTP equipment. The main differences between them include switching core, synchronization, multiservice bearer and transport media.

As is shown in Figure.3, PTN utilizes packetswitched core. Its switching element could be fixedlength or variable-length packets. By contrast, SDH adopts circuit-switched core and its switching element can be VC-12 or VC-4. For synchronization feature, PTN equipment supports both network frequency synchronization and time synchronization while SDH only supports network frequency synchronization. Moreover, PTN can access and bear a variety of services such as TDM, ATM and Ethernet by end-to-end pseudo-wire simulation. PTN also provide MPLS label services and IP service. In addition, PTN utilizes GE/10GE as transport media of transmission side.

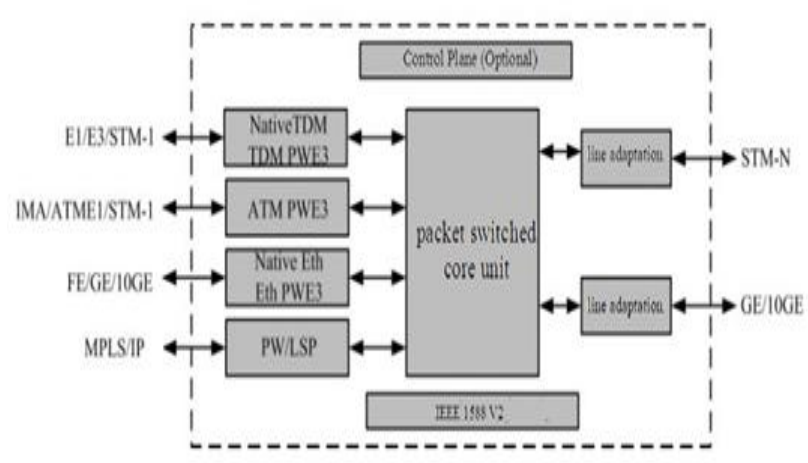

Figure.3 The PTN equipment architecture

Based on the research of PTN technology, the high speed communication network is proposed to support the integrated wide area protection and control. In addition, the development of PTN equipment is founded on the basis of synchronized Ethernet and IEEE 1588 protocol, which also solves the problem of time synchronization and frequency synchronization. Hence PTN technology can fully satisfy all the requirements of IWAPC.

\subsection{Integrated Synchronized Information Platform and Wide Area Power Cloud}

Integrated Synchronized Information Platform The real time synchronized information platform accurately correct wide area information, conduct data mining on the information to investigate the logic relation between the real-time information, to increase the sensitivity, reliability and fault tolerance capability. The real-time information is mainly divided into two types, the electrical information such as the current, voltage and angle, etc.; the state information, such as protection operation and breaker states etc. The former type can correctly reflect the fault, but requires high speed communication, in particular the time synchronization. The information can also include other types, such as the temperature of transformer oil and ambient temperature, wind speed and direction, sunshine intensity etc.

\section{CONCLUSION}

This paper presents an integrated wide area protection and control system based on a hierarchical structure, which integrates protection and control at bay, substation and regional levels. Besides, the proposed high speed synchronized communication network (PTN) and the real-time protection and control information platform is discussed in detail. Based on the proposed entire system, Overall improved performance of protection and control can be expected.

\section{REFERENCES}

[1] Rockerfeller G D, Fault protection with digital computer, IEEE Trans. on PAS, Vol. 88, No.4, April 1969, pp. 438461

[2] Bo Z Q, Jayasinge J A S B, Aggarwal R K, et al. A new scheme for monitoring and protection of power transmission system based on global positioning system, the 31st University Power Engineering Conference, Crete, Greece, 1996: 21-24

[3] Bo Z Q, Weller G, Lomas T, et al. Positional protection of transmission system using global positioning system. IEEE Trans on Power Delivery, 2000, 15 (4): 1163-1168.

[4] Ingelsson B, Lindstrom P O, Karlsson D, et al. Wide-area protection against voltage collapse. IEEE Computer Applications in Power, 1997, 10 (4): 30-35.

[5] Bo Z Q, He J H and Dong X Z. Integrated protection of power network , Relay, 2005, 33 (14): 33-41

[6] Bo Z Q, Zhang B H, Dong X Z, He J H, et al, The development of protection intellectuation and smart relay network, Power System Protection and Control, 2013, 41 (2): 1-12.

[7] Bo Z Q, Wang Lin, Zhou F Q, et al., Substation Cloud Computing for Secondary Auxiliary Equipment, Powercon2014, October 2014, Chengdu, China

[8] Ge S M, Bo Z Q, Zhou F Q, et al, Performance Analysis of Packet Transport Network Communication for Integrated Wide-Area Protection, ICEEA 2015, China 